\section{ORIGINAL RESEARCH}

\author{
C. Mellerio \\ M.-A. Labeyrie \\ F. Chassoux \\ C. Daumas-Duport \\ E. Landre \\ B. Turak \\ F.-X. Roux \\ J.-F. Meder \\ B. Devaux \\ C. Oppenheim
}

\title{
Optimizing MR Imaging Detection of Type 2 Focal Cortical Dysplasia: Best Criteria for Clinical Practice
}

BACKGROUND AND PURPOSE: Type 2 FCD is one of the main causes of drug-resistant partial epilepsy. Its detection by MR imaging has greatly improved surgical outcomes, but it often remains overlooked. Our objective was to determine the prevalence of typical MR imaging criteria for type 2 FCD, to provide a precise MR imaging pattern, and to optimize its detection.

\begin{abstract}
MATERIALS AND METHODS: We retrospectively reviewed 1.5T MR imaging of 71 consecutive patients with histologically proved type 2 FCD. The protocol included millimetric 3D T1-weighted, 2D coronal and axial T2-weighted, and 2D or 3D FLAIR images. Two experienced neuroradiologists looked for 6 criteria: cortex thickening, cortical and subcortical signal changes, blurring of the GWM interface, the "transmantle" sign, and gyral abnormalities. The frequency of each sign and their combination were assessed. We compared the delay between epilepsy onset and surgery, taking into account the time of type 2 FCD detection by MR imaging.
\end{abstract}

RESULTS: Only 42 patients (59\%) had positive MR imaging findings. In this group, a combination of at least 3 criteria was always found. Subcortical signal changes were constant. Three characteristic signs (cortical thickening, GWM blurring, and transmantle sign) were combined in $64 \%$ of patients, indicating that MR imaging can be highly suggestive. However, typical features of type 2 FCD were overlooked on initial imaging in $40 \%$ of patients, contributing to a delay in referral for surgical consideration (17 versus 11.5 years when initial MR imaging findings were positive).

CoNCLUSIONS: A combination of 3 major MR imaging signs allows type 2 FCD to be recognized in clinical practice, thereby enabling early identification of candidates for surgery.

ABBREVIATIONS: EEG = electroencephalography; ${ }^{18} \mathrm{FDG}=$ fluorine 18 FDG; GWM = gray-white matter; type 2 FCD $=$ type 2 focal cortical dysplasia

$\mathbf{T}_{\mathrm{r}}$ ype 2 FCD is one of the main causes of extratemporal drugresistant partial epilepsy that is surgically curable. It corresponds to Taylor-type focal cortical dysplasia, according to recent classifications, ${ }^{1,2}$ a more homogeneous pathologic entity than other subtypes of cortical dysplasia, especially type 1 FCD. The major predictor of a favorable surgical outcome is complete removal of the dysplastic cortex, ${ }^{3-7}$ and accurate presurgical assessment of the lesion extent is crucial to improve surgical results. During the past decade, the development of MR imaging protocols specifically designed for type 2 FCD detection has contributed to the steady increase of candidates for surgery and to a favorable outcome, with a remission rate of up to $90 \%$ when MR imaging findings are positive compared with $40 \%-60 \%$ when MR imaging findings are negative. ${ }^{3,6-9}$ The MR imaging diagnosis remains difficult, however, even in specialized centers by using appropriate protocols, with up to $50 \%$ of type 2 FCD cases remaining undetected or being diagnosed late, thereby depriving patients of effective treatment for years. ${ }^{3}$ Despite severe and intractable

Received December 18, 2011; accepted after revision January 25, 2012

From the Departments of Neuroimaging (C.M., M.-A.L., J.-F.M., C.O.), Neurosurgery (F.C., E.L., B.T., F.-X.R., B.D.), and Neuropathology (C.D.-D.), Centre Hospitalier Sainte-Anne, Paris, France; and Université Paris Descartes (C.M., M.-A.L., F.C., J.-F.M., C.O.), Paris, France.

Please address correspondence to Charles Mellerio, MD, Service d'Imagerie Morphologique et Fonctionnelle, Centre Hospitalier Sainte-Anne, 1 rue Cabanis 75014-Paris; e-mail: c.mellerio@ch-sainte-anne.fr

http://dx.doi.org/10.3174/ajnr.A3081 epilepsy, the average time from onset of seizures to surgery is around 14 years in most reported series. ${ }^{3,10-12}$

Typical MR imaging features have previously been described. ${ }^{3,6,7,13-21}$ These include abnormalities of the cortex (thickening, T2 signal increase, gyral abnormalities) and of the subcortical white matter (blurring of the GWM interface, T2 signal increase, "transmantle" sign). Initial MR imaging descriptions relied on a limited sample of patients. ${ }^{7,15}$ However, more recent larger series do not provide a comprehensive analysis of all reported MR imaging signs and the prevalence of each sign varies considerably between studies (Table 1). In addition, the proportion of negative MR imaging findings in type 2 FCD differs widely, likely because of differences in imaging protocols and selection bias.

We performed a retrospective study on a large cohort of surgically treated patients with type 2 FCD investigated with high-resolution MR imaging during the past decade. Our goal was to determine the prevalence of each previously described MR imaging criterion for type 2 FCD and to define a precise MR imaging pattern so as to optimize its detection in clinical practice. Our second goal was to determine the influence of MR imaging positivity on delay to surgical referral.

\section{Materials and Methods}

\section{Patients}

We retrospectively reviewed the MR imaging data of 71 consecutive patients (40 males, 24 children) with a histologic diagnosis of type 2 FCD who underwent surgery for intractable epilepsy between May 


\begin{tabular}{|c|c|c|c|c|c|c|c|c|c|}
\hline & $\begin{array}{l}\text { Urbach et al, } \\
2002^{7}\end{array}$ & $\begin{array}{c}\text { Colombo et al, } \\
2003^{15}\end{array}$ & $\begin{array}{l}\text { Widdess- } \\
\text { Walsh et al, } \\
2005^{14}\end{array}$ & $\begin{array}{c}\text { Besson et al, } \\
2008^{26}\end{array}$ & $\begin{array}{c}\text { Widjaja et al, } \\
2008^{28}\end{array}$ & $\begin{array}{c}\text { Kim et al, } \\
2009^{4}\end{array}$ & $\begin{array}{l}\text { Krsek et al, } \\
2009^{6}\end{array}$ & $\begin{array}{l}\text { Lerner et al, } \\
2009^{3}\end{array}$ & $\begin{array}{l}\text { This } \\
\text { Study }\end{array}$ \\
\hline Period of study & $1996-2000$ & 1996-2000 & 1990-2002 & NA & $1991-2005$ & 1995-2005 & $2002-2005$ & $2000-2007$ & $2000-2011$ \\
\hline No. of patients & 22 & 15 & 48 & 26 & 13 & 28 & 16 & 64 & 71 \\
\hline $\begin{array}{l}\text { Negative findings } \\
\text { on MRI }\end{array}$ & 0 & $5(33 \%)$ & $10(21 \%)$ & 0 & $2(15 \%)$ & $6(21 \%)$ & 0 & 1 & $29(41 \%)$ \\
\hline Cortical thickening & $18(81 \%)$ & $10(67 \%)$ & NA & NA & $8(61 \%)$ & NA & $8(50 \%)$ & $43(67 \%)$ & $30(71 \%)$ \\
\hline $\begin{array}{l}\text { Blurring of GWM } \\
\text { junction }\end{array}$ & $8(36 \%)$ & $13(87 \%)$ & NA & NA & $11(84 \%)$ & NA & $11(69 \%)$ & $47(73 \%)$ & $38(90 \%)$ \\
\hline $\begin{array}{l}\text { Cortical signal } \\
\text { changes }\end{array}$ & NA & $6(40 \%)$ & NA & NA & $2(15 \%)$ & NA & $10(62 \%)$ & $22(35 \%)$ & $21(50 \%)$ \\
\hline $\begin{array}{l}\text { Subcortical signal } \\
\text { changes }\end{array}$ & $22(100 \%)$ & $13(87 \%)$ & $27(56 \%)$ & NA & $11(84 \%)$ & NA & $14(87 \%)$ & $64(100 \%)$ & 42 (100\%) \\
\hline Transmantle sign & $18(81 \%)$ & $3(20 \%)$ & NA & $5(19 \%)$ & $8(72 \%)$ & NA & $6(37 \%)$ & NA & $35(83 \%)$ \\
\hline Sulcal abnormalities & NA & NA & NA & NA & $3(23 \%)$ & NA & 11 (69\%) & NA & $22(52 \%)$ \\
\hline Atrophy & NA & $3(20 \%)$ & NA & NA & $2(15 \%)$ & NA & $7(44 \%)$ & $11(17 \%)$ & 0 \\
\hline
\end{tabular}

Note:-NA indicates not available.

2000 and May 2011. Presurgical evaluation included ictal video-EEG, high-resolution MR imaging, functional MR imaging, and ${ }^{18} \mathrm{FDG}-$ PET scans for all patients and stereo-EEG for 43 of them $(60 \%)$. Median age was 6 years (range, 1-20 years) at epilepsy onset and 20 years (range, $8-52$ years) at surgery. No patient had clinical criteria for tuberous sclerosis. The study was approved by the Ethics Committee of Ile de France III and was found to conform to generally accepted scientific principles and ethical standards.

\section{MR Imaging Acquisition}

Brain MRI was performed on a $1.5 \mathrm{~T}$ MR imaging scanner (Signa Excite; GE Healthcare, Milwaukee, Wisconsin) and included the following sequences: a volumetric gradient-echo T1-weighted inversion recovery acquisition (section thickness $=1.2 \mathrm{~mm}, \mathrm{FOV}=240 \times 240$ $\mathrm{mm}, 256 \times 192$ matrix, NEX $=1$ ), coronal and axial 2D fast spinecho T2-weighted acquisitions (thickness $=4 \mathrm{~mm}$, no gap, FOV $=$ $240 \times 240 \mathrm{~mm}, 512 \times 256$ matrix, NEX $=2$ ), FLAIR by using $2 \mathrm{D}$ contiguous sections (thickness $=5 \mathrm{~mm}$, FOV $=240 \times 240 \mathrm{~mm}$, matrix $=256 \times 192, \mathrm{NEX}=1$ ), or a $3 \mathrm{D}$ acquisition (section thickness $=1.2 \mathrm{~mm}$, FOV $=240 \times 240 \mathrm{~mm}, 256 \times 226$ matrix, NEX $=1$ ). A 3D T1 fast gradient-echo acquisition after injection of gadolinium was also performed; this was not part of the epilepsy presurgical standard protocol but was needed for accurate identification of vascular landmarks with neuronavigational guidance during intracranial recordings or surgery.

\section{Data Analysis}

Two neuroradiologists ( 7 and 9 years' experience), aware of the final localization of the operated lesion, retrospectively reviewed all MRI in consensus to look for structural abnormalities. They analyzed 6 criteria: 1) abnormal cortical thickness, defined as a thickening (or thinning) of at least $50 \%$ of the normal cortex, visible on T1WI and T2WI sequences, on at least 2 orthogonal planes, to rule out partial volume effects (when visible on 1 sequence only, it was defined as a "pseudothickening"); 2) abnormal cortical signal intensity on T1WI, T2WI, and/or FLAIR, defined as signal changes involving the entire thickness of the cortex; 3 ) blurring of the GWM junction, visible on at least 1 sequence and in 2 orthogonal planes; and 4) abnormal signal intensity of subcortical white matter relative to normal cortex (this latter abnormality was further classified as either "marked," when the subcortical white matter signal was at least identical to that of the normal cortex, or "subtle" otherwise (Fig 1); 5) abnormal signal intensity of deep white matter, the so-called transmantle sign, defined as a subcortical white matter signal-intensity change, tapering toward the ventricle on T1WI and/or T2WI (Fig 1); ${ }^{22}$ and 6) a major abnormality of sulcal morphology, defined as an abnormality of the depth, angulation, or shape of a sulcus, compared with the contralateral side, on reformatted 3D T1WI or on T2WI sequences.

The presence of calcifications, cysts, contrast enhancement, atrophy or mass effect, and vascular abnormalities was also recorded. MR imaging was considered negative for the diagnosis of type 2 FCD when none of the above abnormalities were present and positive when at least 1 of the $6 \mathrm{MR}$ imaging criteria was present. In addition, we looked for minor sulcal abnormalities in patients with negative MR imaging finding (ie, unusual sulci patterns not discernible enough to be classified as major). Because these findings were regarded as nonspecific or doubtful, MR imaging findings were still considered negative.

To determine the influence of MR imaging positivity on referring the patient for a presurgical work-up, we compared the time from epilepsy onset to surgery between the groups with positive and negative MR imaging findings by using a Wilcoxon test. In addition, in the subgroup of patients with positive MR imaging findings, we reviewed all previous MRI with negative findings available in the medical records to retrospectively detect the lesion and compared the time from epilepsy onset to surgery with that of patients whose first MR imaging findings were considered positive, by using a Wilcoxon test.

\section{Results}

Among the 71 patients, type 2 FCD was located in the frontal lobe ( $n=60$, including 28 in the central region limited by the pre- and postcentral sulci and 3 in the insula); parietal lobe $(n=7)$; occipital lobe $(n=2)$; or temporal $(n=2)$ lobe. All lesions were limited to a single lobe and were unilateral, with a right/left hemisphere ratio of 1:3. Histologic confirmation of type 2 FCD was based on a focal disorganization of the cortical cytoarchitecture with giant dysmorphic neurons. Balloon cells were present in 63 patients (type 2b FCD) and were not found in the cortical specimen in the remaining patients (type $2 \mathrm{a}$ FCD). 

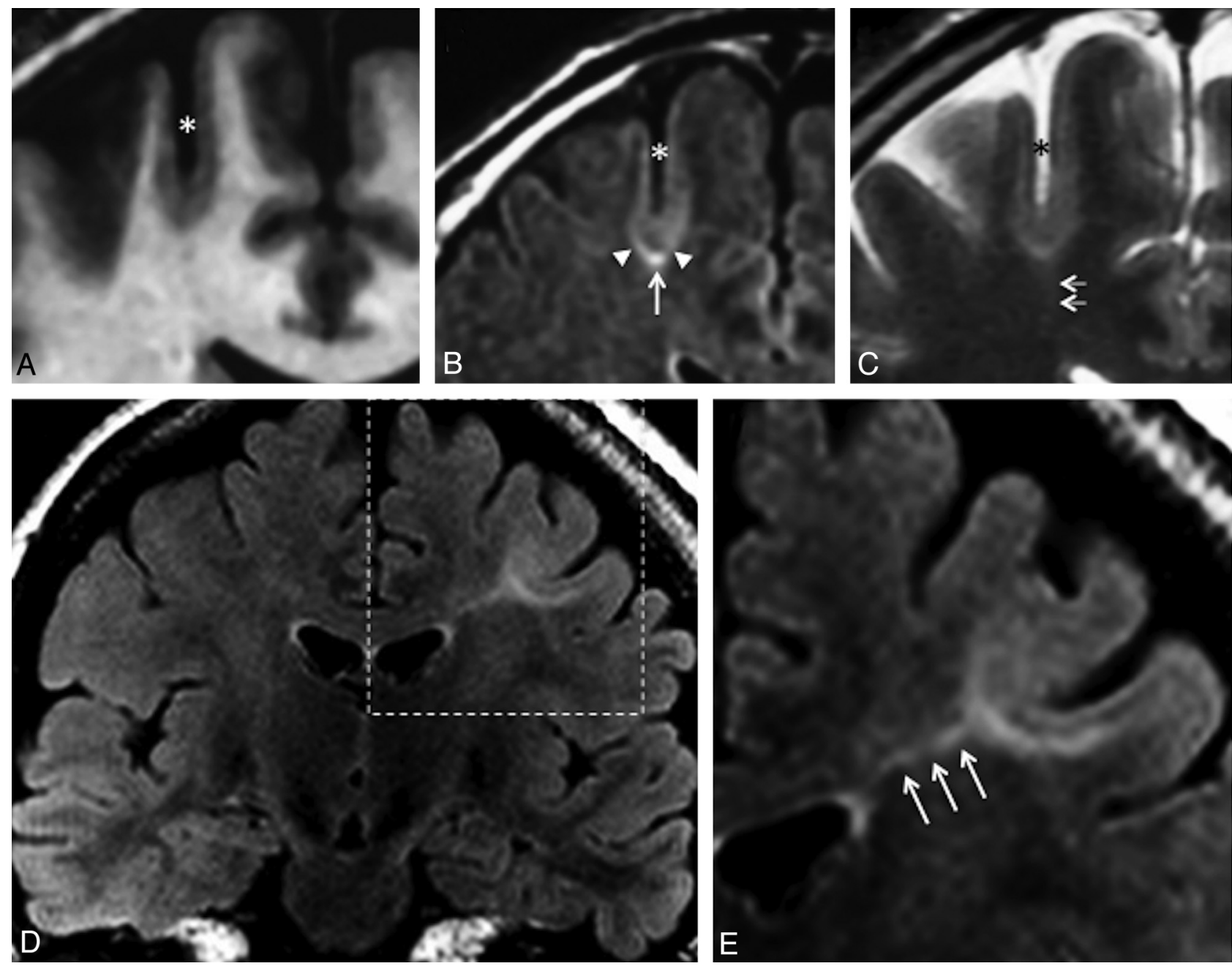

Fig 1. Typical MR imaging signs of type 2 FCD. Coronal 3D T1WI (A), coronal FLAIR (B), and coronal T2WI (C) in a 45-year-old man with left motor seizures, epilepsy onset at 6 years, and findings on several previous MR images considered normal. An unusually deep and straight sulcus in the precentral region (asterisk), with minimal cortical thickening at the bottom of the sulcus, and cortical signal increase in T2WI and FLAIR. Abnormal subcortical signal, marked at the bottom (arrow), surrounded by an area of subtle signal increase (arrowheads), responsible for a gradient of signal abnormalities from the periphery to the center of the dysplasia. Barely perceptible transmantle sign $(C$, double arrows). Coronal FLAIR $(D)$ and magnification $(E)$ of the left central region in a 19-year-old woman with right motor seizure, epilepsy onset at 16 years of age. These MR images show a marked increased signal, tapering gradually from the gray-white matter interface to the superolateral edge of the lateral ventricle (triple arrows), typical of a transmantle sign.

\section{Positive Findings on MR Imaging}

Forty-two patients (59\%) had positive MR imaging findings. Twelve patients (29\%) had all 6 criteria. All patients had at least 3 of the 6 criteria. All patients had either cortical thickening, blurring of GWM interface, or transmantle sign; 27 patients $(64 \%)$ had all 3 of these signs.

Cortical thickening was present in 30 patients ( $71 \%$ of positive MR imaging findings), did not exceed twice the normal cortex, and was limited to a small cortical area. Seven other patients (17\%) had "pseudothickening," visible on only 1 of the sequences. This "pseudothickening" was related to signal changes of the subcortical white matter (Fig 2).

Cortical signal changes were present in 21 patients $(50 \%$ of MR imaging with positive findings). These consisted of hyperintensity on T2WI in 15 patients (best seen on FLAIR sequences), hyperintensity on T1WI in 2 patients, and increased signal on both sequences in the remaining 4 patients. These were located at the bottom of the dysplastic sulcus, variably spreading to the surrounding gyri.

Blurring of the GWM interface was present in 38 patients
(90\% those with positive MR imaging findings). It was colocated with cortical thickening, when present, in all patients except 1 . As with cortical thickening, this sign could be obvious on 1 sequence and barely visible on others (Fig 2).

Abnormal signal intensity of subcortical white matter was seen in all patients with positive MR imaging findings. It was "marked" in 31 patients (73\%). "Subtle" changes were not visible on T1WI. Most interesting, in 13 of the 31 patients with marked subcortical increased signal, this abnormality extended from the depth of the sulcus to the surrounding white matter, with a symmetric gradual centrifugal signal decrease (Fig 1).

The transmantle sign was present in 35 patients $(83 \%$ of MR imaging with positive findings) and was typically mildly hyperintense on T2WI and FLAIR and hypointense in T1WI. It spread along the axis of the abnormal sulcus, running perpendicular toward the wall of the lateral ventricle (Fig 1). Its thickness was proportional to the width of the subcortical abnormalities.

Major sulcal abnormalities were present in 22 patients ( $52 \%$ of those with positive MR imaging findings). These con- 

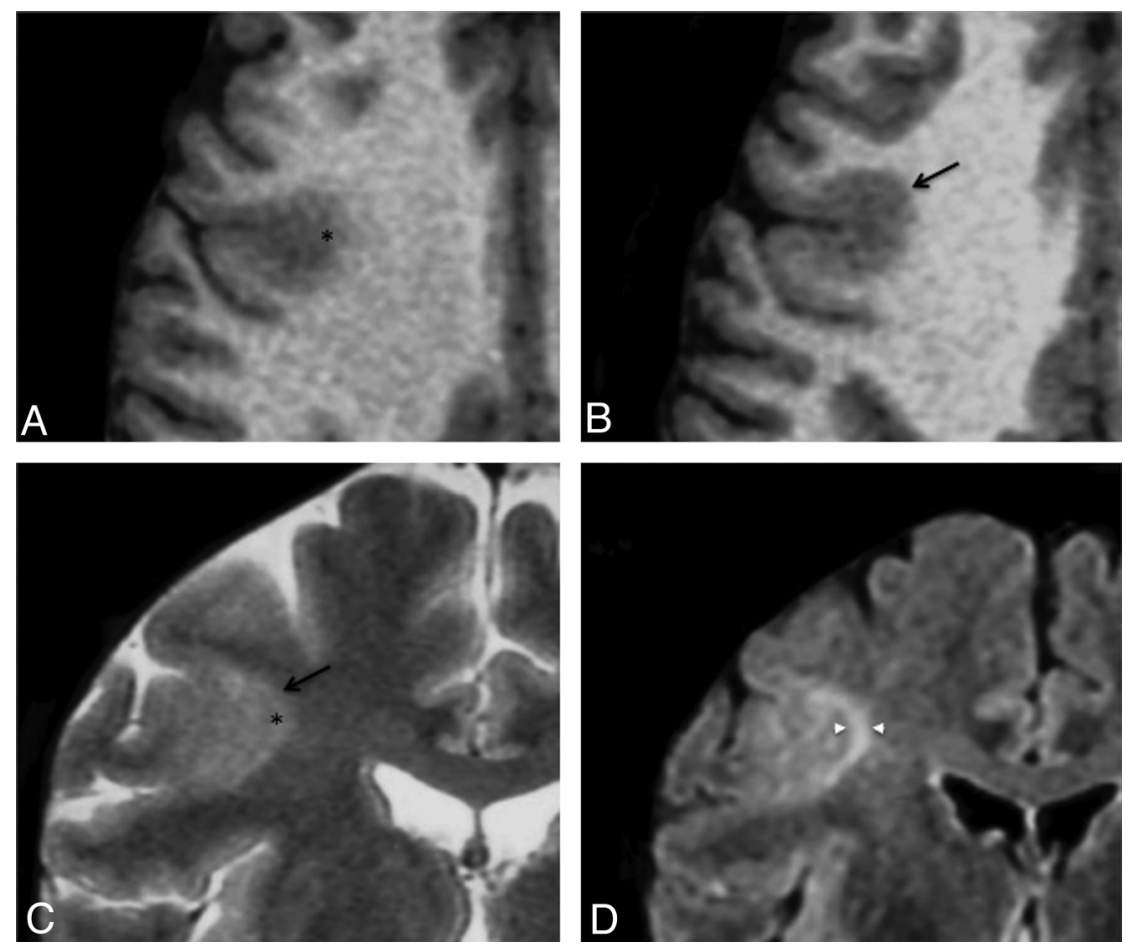

Fig 2. Cortical pseudothickening. Axial T1WI $(A$ and $B)$ and coronal T2WI $(C)$ in a 29-year-old man with left motor seizures, onset at 3 years of age. These images show cortical thickening (arrow) and blurring (asterisk) of the gray-white matter interface of the right central sulcus. D, Coronal FLAIR image allowing cortical to be distinguished from subcortical signal increase (arrowheads).

sisted of sulci that were unusually deep $(n=15)$, wide $(n=5)$, and/or with unusual angulation $(n=11)$ (Fig 1). Such sulcal abnormalities were never isolated but were associated with other typical type 2 FCD features.

Microcalcifications were observed in the periventricular region in a patient with a large dysplasia in the temporal lobe. A developmental venous abnormality adjacent to a frontal dysplasia was seen in another patient. A small $(<10 \mathrm{~mm})$ cystic component was present in 3 patients. Parenchymal contrast enhancement, focal atrophy or mass effect, complete disappearance of the GWM interface, or marked thinning of the cortex was never observed.

Among the 42 patients with positive MR imaging findings, 16 had at least 1 brain MR imaging finding that had been considered normal during the course of epilepsy. In all 16 cases, the lesion was retrospectively visible. The delay from epilepsy onset to surgery was $>5$ years longer in patients with negative initial MR imaging (median, 17 years; range, 6-42 years) than in those whose first MR imaging findings were positive (11.5 years; range, 1-29 years), though not reaching significance due to the small number of patients $(P=.06)$.

\section{Negative Findings on MR Imaging}

Twenty-nine patients (41\%) had negative MR imaging findings (Fig 3), despite histologic confirmation of type 2 FCD (Table 2). For these patients, FDG-PET findings were positive in $25(86 \%)$ cases, showing a focal or regional hypometabolism, contributing to the detection of the lesion. In addition, intracranial recording by using depth electrodes (stereo-EEG) was used to determine the epileptogenic zone and the extent of the cortical resection. In 4 patients, intracranial recording was deemed not necessary because PET demonstrated a focal hy- pometabolism corresponding to a single gyrus, highly suggestive of a focal lesion.

Moreover, in 13 cases of negative MR imaging findings, minor sulcal abnormalities were observed in the vicinity of type 2 FCD (Fig 4), sometimes not strictly co-localized with the dysplastic lesion. As with major sulcal abnormalities, minor features corresponded to the unusual depth, width, or shape of the sulcus but were not clear enough to be considered abnormal. In addition, none of the other criteria suggestive of type 2 FCD were found in these cases, in contrast to the major sulcal abnormalities described in cases with positive MR imaging findings, which were never isolated (Fig 1).

Balloon cells were present in $79 \%$ of the cases with negative MR imaging findings and with similar proportion in patients with minor sulcal abnormalities (10 of 13) than in those with strictly normal MR imaging findings (13 of 16).

The delay from epilepsy onset to surgery was shorter in the patients with negative MR imaging findings (median, 11.5 years; range, $1-31$ years) than in the group with positive MR imaging findings ( 16 years; range, $1-42$ years) $(P=.03)$. This apparently surprising finding is related to a bias of recruitment, with a high proportion of children with negative MR imaging findings and short epilepsy duration due to special collaboration with pediatric teams and systematic use of FDGPET in this population.

\section{Discussion}

The main findings of this systematic qualitative MR imaging analysis in a series of 71 consecutive patients with histologically confirmed type 2 FCD are as follows: 1) Only 59\% had positive MR imaging findings despite optimal MR imaging techniques according to recommended guidelines; ${ }^{23} 2$ ) all pa- 

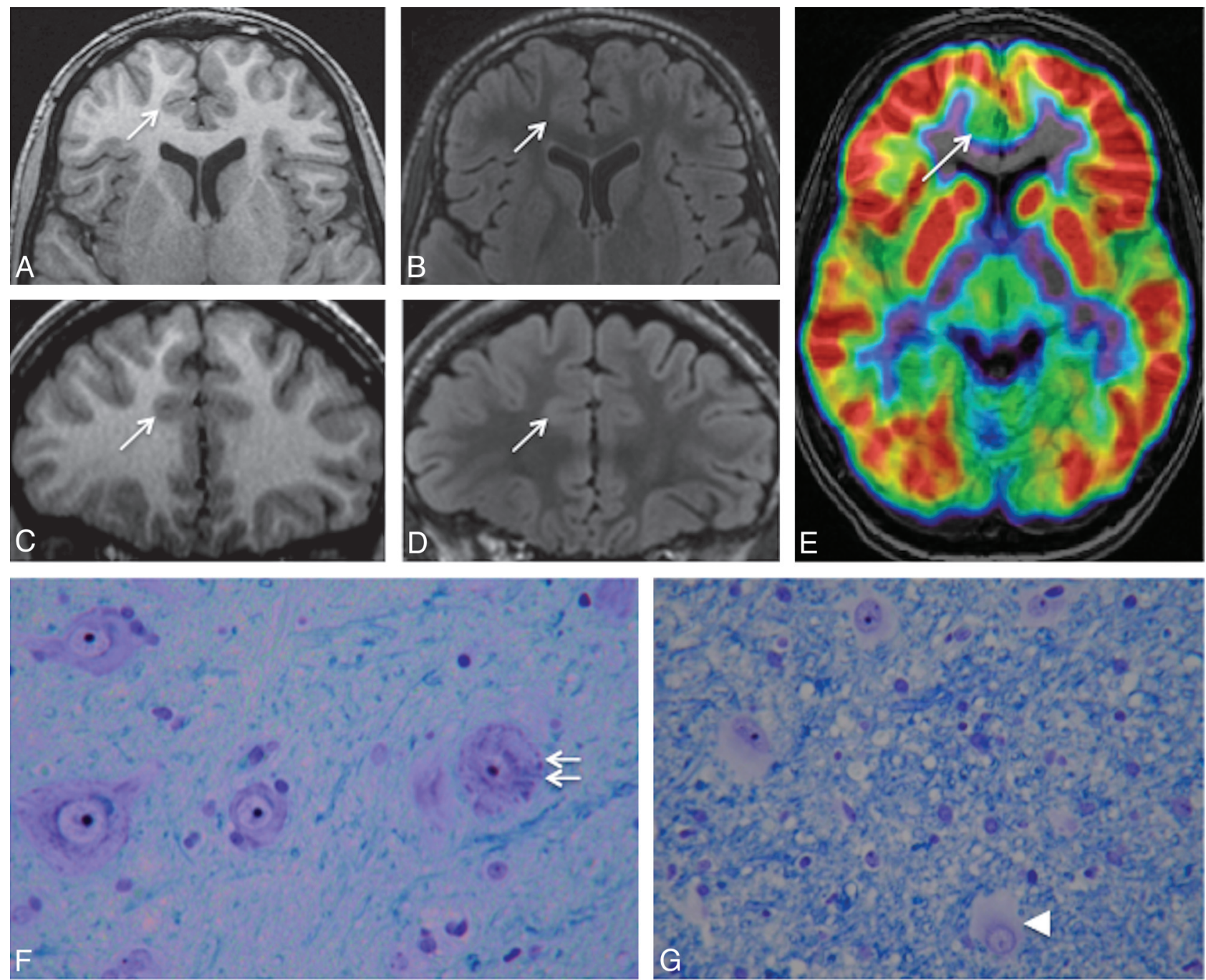

Fig 3. Negative MR imaging findings. Axial and coronal 3D T1WI $(A$ and $C)$ and axial and coronal 3D FLAIR ( $B$ and $D)$ in a 15-year-old boy with right frontal lobe epilepsy, nocturnal seizure predominance, and onset at 12 years. Absence of the 6 criteria and no minor sulcal abnormality were found. $E_{,}{ }^{18} \mathrm{FDG}$-PET coregistered on MR imaging (axial section) allows recognition of a gyral hypometabolism corresponding to the anterior part of the right cingulate cortex (arrow). $F$ and $G$, Histology slides show typical features of type $2 \mathrm{~b}$ FCD with giant dysmorphic neurons in the cortex (double arrow) and balloon cells in the underlying white matter (arrowhead) (Klüver-Barrera, original magnification $\times 40$ ).

Table 2: Clinical and histologic data of patients with positive and negative findings on MRI

\begin{tabular}{lccc}
\hline & $\begin{array}{c}\text { Negative } \\
\text { MRI } \\
\text { Findings }\end{array}$ & $\begin{array}{c}\text { Positive } \\
\text { MRI } \\
\text { Findings }\end{array}$ & Total \\
\hline No. of patients & 29 & 42 & 71 \\
Sex ratio (M/F) & 0.8 & 1.8 & 1.3 \\
Age at onset (yr) (median) & $6(1-20)$ & $5.8(1-20)$ & $6(1-20)$ \\
$\quad$ (range) & $17(8-41)$ & $22.5(8-52)$ & $20(8-52)$ \\
Age at surgery (yr) (median) & & & \\
$\quad$ (range) & 1.4 & 1.25 & 1.35 \\
Location of FCD & 23 & 37 & 60 \\
Left/right ratio & 4 & 3 & 7 \\
Frontal lobe & 1 & 1 & 2 \\
Parietal lobe & 1 & 1 & 2 \\
Temporal lobe & & & \\
$\quad$ Occipital lobe & 6 & 2 & 8 \\
Type 2 FCD subtypes & 23 & 40 & 63 \\
$\quad$ Type 2a (no balloon cells) & & & \\
Type 2b (balloon cells) & &
\end{tabular}

tients with positive MR imaging findings presented with least 3 of the 6 recognized MR imaging criteria for type 2 FCD, with combined cortical thickening, blurring of the GWM interface, and the transmantle sign in $64 \%$ of patients; and 3) MR imaging features suggestive of type 2 FCD were overlooked on initial imaging in $40 \%$ of the cases, leading to late referral for surgical consideration.

Cortical thickening and blurring of the GWM demarcation are considered to be major signs, corresponding to the presence of dysmorphic neurons and balloon cells in the cortex and GWM junction, ectopic neurons, or axonal loss in white matter. ${ }^{7,8,14,15}$ We additionally found subtle pseudothickening (ie, a subtle subcortical signal increase similar to that of the cortex) in a few patients, contrasting with the extensive thickening reported in previous studies..$^{3,7,11,15}$ The subtlety of this sign is in line with histologic data showing that cortical thickening is more focal and less obvious than previously described. ${ }^{24,25}$ Cortical thickening has been the focus of an expert consensus, stipulating that it must be seen in 2 adjacent 

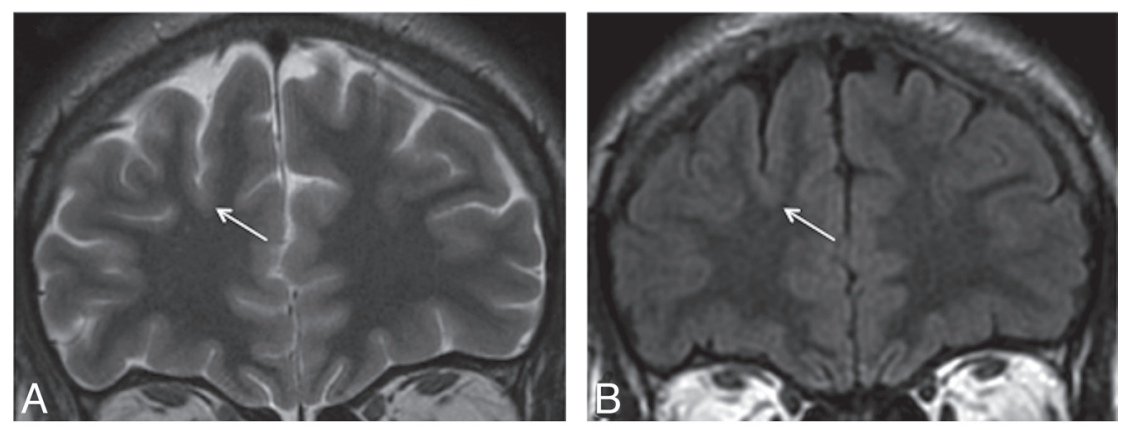

Fig 4. Negative $M R$ imaging findings with minor sulcal abnormalities. Coronal T2WI $(A)$, FLAIR $(B)$, and T1WI $(C)$ in a 36-year-old man with right nocturnal frontal lobe epilepsy. onset at 20 years of age. None of the 6 criteria were found. A minor sulcal abnormality is perceptible in the right superior frontal area, with an unusually large and deep sulcus (arrow).

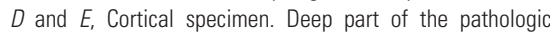
sulcus with typical type 2a FCD features: cortical disorganization and the presence of giant neurons (arrowhead) without balloon cells. Note that the good delineation of the graywhite matter interface (double arrow) is correlated with the absence of blurring on MR imaging (Klüver-Barrera, original magnification $\times 5[D] ; \times 15[E])$.
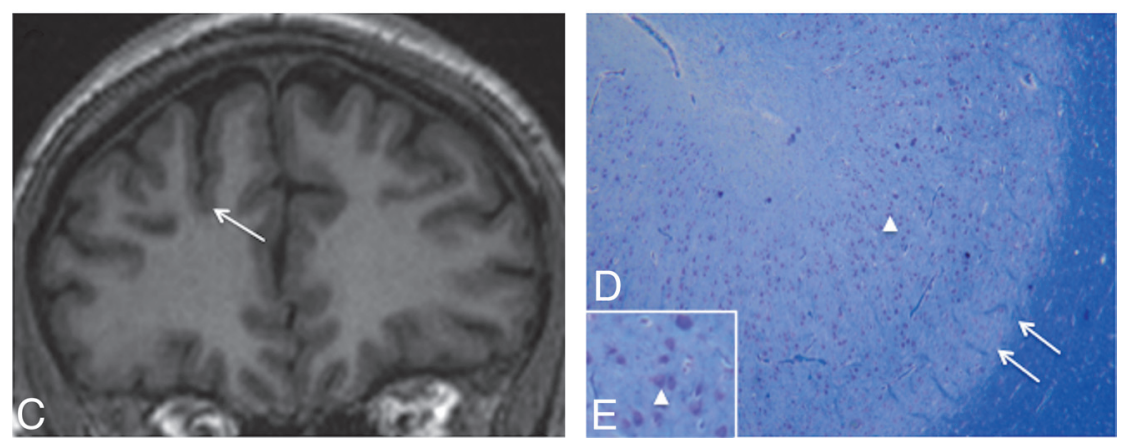

sections and with 2 different pulse weightings, to avoid being confounded with pseudothickening. ${ }^{25}$ We did not observe any sign of lobar, gyral, or focal cortical atrophy, in contrast to others who reported atrophy in $15 \%-44 \%$ of cases (Table 1). This subtle and subjective sign, more common in type 1 focal cortical dysplasia, ${ }^{6}$ may have been underestimated in our analysis. It is also possible that what we considered a deep dysplastic sulcus was interpreted by others as pseudofocal atrophy. ${ }^{26}$ We never observed any major cortical thinning as encountered in ischemic or traumatic sequelae.

The third major sign consisted of subcortical white matter abnormalities, which were found in all patients with positive MR imaging findings. This is likely due to the clear-cut signal changes on T2WI or FLAIR. Contrary to the authors of a previous report, ${ }^{16}$ we found no correlation between the presence of balloon cells and subcortical signal abnormalities (Table 2).

The signal increase of the cortex is a well-known sign of type 2 FCD, though rarely emphasized. ${ }^{3,14}$ It was found in $48 \%$ of patients with positive MR imaging findings, within the $15 \%-62 \%$ range of previous reports (Table 1). ${ }^{3,6,13,15}$ It was often moderate and was more clearly seen on FLAIR sequences. This abnormality could be related to a high attenuation of balloon cells in the cortex. ${ }^{14}$ With the increased use of 3D FLAIR and high-field MR imaging, this sign may, in the future, be easier to detect and become more reliable.

The transmantle sign is reported to be a specific feature for malformations of cortical development. ${ }^{22}$ This typical pattern overlaps the path of migrating neuroblasts, consistent with a disruption of early corticogenesis. It has been related to the presence of balloon cells and hypomyelination in the white matter underlying the dysplastic lesion. ${ }^{7,22}$ This sign may also occur in other developmental abnormalities, such as venous or arteriovenous malformations ${ }^{27}$ and, when isolated, is not specific for type 2 FCD. However, its association with cortical thickening and GWM blurring provides the most reliable pattern for the diagnosis of type 2 FCD. We observed the transmantle sign in $>80 \%$ of patients with positive MR imaging findings, which is a higher proportion than that reported in even the most recent studies. In 2 recent studies, the frequency of the transmantle sign reached $60 \%,{ }^{7,28}$ whereas its frequency did not exceed $30 \%$ in other studies, suggesting that it may have been underestimated in earlier reports. $6,7,13,15,26$

Abnormalities of sulcal morphology were present in nearly one-half of the cases with positive MR imaging findings. Such features are difficult to assess and are likely underestimated. Isolated minor sulcal abnormalities may also be retrospectively found in cortical areas containing a small type 2 FCD, as observed in nearly half of the cases with negative MR imaging findings (Fig 4). Sulcal abnormalities have already been described $^{29}$ but have received little attention, and their prevalence has never been evaluated. Only 1 study $^{26}$ confirmed by quantitative analysis that small type 2 FCDs were preferentially located at the bottom of an abnormally deep sulcus. Their physiologic mechanisms have not been elucidated to date. Nevertheless, most malformations of cortical development are associated with abnormal gyral/sulcal morphology, suggesting that the organization of sulci is intimately linked to the early stages of cortical development. ${ }^{30}$

Another striking finding in this study is that abnormalities were arranged symmetrically relative to an axis perpendicular to the cortex. Of note, the axis of symmetry overlapped the trajectory of the transmantle sign. In addition, in half of the cases, a gradient of decreasing abnormal signal intensity from the bottom of the dysplastic sulcus to the surrounding gyri was found (Fig 1). This finding confirms that irrespective of the size of the lesion, maximum cellular abnormalities are located deep in the sulcus.

The proportion of patients with type 2 FCD with negative MR imaging findings (41\%) was higher than that in most reported series ${ }^{3,6-8,13-15,26}$ but similar to the rate reported using stereo-EEG as a diagnostic tool. ${ }^{31}$ This is likely explained by the fact that in our patients with negative MR imaging findings, surgery was based on a combination of FDG-PET and stereo-EEG. Balloon cells were found in the cortical specimens 
of $79 \%$ of our patients with negative MR imaging findings (Table 2), in contrast to previous reports suggesting that balloon cell FCDs (type 2b) are generally characterized by signal changes in the white matter. ${ }^{16}$

Finally, the localization of type 2 FCD was overwhelmingly frontal and rarely temporal in our series, as in others, ${ }^{8,14,15}$ whereas other epileptogenic lesions are predominantly located in the temporal lobe. This suggests that frontal drug-resistant partial epilepsy with normal MR imaging findings should raise the suspicion of type 2 FCD, and this is supported by surgical series of cryptogenic partial epilepsy, in which up to $40 \%$ of the resected cortical specimens (especially in the frontal lobe) corresponded to type 2 FCD at histology. ${ }^{9,31,32}$ Moreover, a recent report of the International League against Epilepsy noted that none of the children with epilepsy diagnosed with tumor or ischemia had normal MR imaging findings. ${ }^{11}$

Our study has some limitations. It was retrospective and was based solely on patients who underwent surgery and thus does not represent the entire spectrum of type 2 FCD. Nevertheless, the study of unoperated patients would be limited by the lack of histologic confirmation. Furthermore, due to the lack of a control group, we cannot assess the specificity of the MR imaging abnormalities, especially the transmantle sign. In addition, positive MR imaging findings (59\% of our patients) were based on conventional visual analysis. Voxel-based postprocessing methods proved a significant benefit in comparison with visual analysis alone $e^{33,34}$ and would possibly decrease our rate of negative MR imaging findings. However, these techniques apply algorithms not applicable in routine practice. Furthermore, the feature maps direct the attention to suspicious regions, but the interpretation still requires an experienced reader to confirm, with conventional MR imaging, the presence of a type 2 FCD. Finally, the use of a 3T magnetic field could have increased the number of positive findings on MRI as reported in a recent study. ${ }^{35}$

\section{Conclusions}

We emphasize that nearly $60 \%$ of type 2 FCDs may be recognized on 1.5T MR imaging and propose that a combination of features (found in two-thirds of the cases), comprising focal cortical thickening, GWM blurring, and the transmantle sign, is highly suggestive of this FCD subtype. Early identification of this lesion is crucial to minimize the delay in referring patients for surgical consideration.

Disclosures: Baris Turak-UNRELATED: Travel/Accommodations/Meeting Expenses Unrelated to Activities Listed: Cyberonics, Comments: traveling expenses to epilepsy conferences in Rome and Bordeaux paid by Cyberonics.

\section{References}

1. Blumcke I, Thom M, Aronica E, et al. The clinicopathologic spectrum of focal cortical dysplasias: a consensus classification proposed by an ad hoc Task Force of the ILAE Diagnostic Methods Commission. Epilepsia 2011;52:158-74

2. Palmini A, Najm I, Avanzini G, et al. Terminology and classification of the cortical dysplasias. Neurology 2004;62(suppl 3):S2-8

3. Lerner JT, Salamon N, Hauptman JS, et al. Assessment and surgical outcomes for mild type I and severe type II cortical dysplasia: a critical review and the UCLA experience. Epilepsia 2009;50:1310-35

4. Kim DW, Lee SK, Chu K, et al. Predictors of surgical outcome and pathologic considerations in focal cortical dysplasia. Neurology 2009;72:211-16

5. Chassoux F, Devaux B, Landre E, et al. Stereoelectroencephalography in focal cortical dysplasia: a 3D approach to delineating the dysplastic cortex. Brain 2000;123:1733-51
6. Krsek P, Maton B, Jayakar P, et al. Incomplete resection of focal cortical dysplasia is the main predictor of poor postsurgical outcome. Neurology 2009;72: 217-23

7. Urbach H, Scheffler B, Heinrichsmeier T, et al. Focal cortical dysplasia of Taylor's balloon cell type: a clinicopathological entity with characteristic neuroimaging and histopathological features, and favorable postsurgical outcome. Epilepsia 2002;43:33-40

8. Tassi L, Colombo N, Garbelli R, et al. Focal cortical dysplasia: neuropathological subtypes, EEG, neuroimaging and surgical outcome. Brain 2002;125(pt 8):1719-32

9. Jeha LE, Najm I, Bingaman W, et al. Surgical outcome and prognostic factors of frontal lobe epilepsy surgery. Brain 2007;130:574-84

10. Salamon N, Kung J, Shaw SJ, et al. FDG-PET/MRI coregistration improves detection of cortical dysplasia in patients with epilepsy. Neurology 2008;71: $1594-601$

11. Widdess-Walsh P, Diehl B, Najm I. Neuroimaging of focal cortical dysplasia. J Neuroimaging 2006;16:185-96

12. Chassoux F, Rodrigo S, Semah F, et al. FDG-PET improves surgical outcome in negative MRI Taylor-type focal cortical dysplasias. Neurology 2010;75: $2168-75$

13. Widjaja E, Nilsson D, Blaser S, et al. White matter abnormalities in children with idiopathic developmental delay. Acta Radiol 2008;49:589-95

14. Widdess-Walsh P, Kellinghaus C, Jeha L, et al. Electro-clinical and imaging characteristics of focal cortical dysplasia: correlation with pathological subtypes. Epilepsy Res 2005;67:25-33

15. Colombo N, Citterio A, Galli C, et al. Neuroimaging of focal cortical dysplasia: neuropathological correlations. Epileptic Disord 2003;5(suppl 2):S67-72

16. Chan S, Chin SS, Nordli DR, et al. Prospective magnetic resonance imaging identification of focal cortical dysplasia, including the non-balloon cell subtype. Ann Neurol 1998;44:749-57

17. Kuzniecky R, Morawetz R, Faught E, et al. Frontal and central lobe focal dysplasia: clinical, EEG and imaging features. Dev Med Child Neurol 1995; 37:159-66

18. Lee SK, Choe G, Hong KS, et al. Neuroimaging findings of cortical dyslamination with cytomegaly. Epilepsia 2001;42:850-56

19. Mackay MT, Becker LE, Chuang SH, et al. Malformations of cortical development with balloon cells: clinical and radiologic correlates. Neurology 2003;60:580-87

20. Bernasconi A, Antel SB, Collins DL, et al. Texture analysis and morphological processing of magnetic resonance imaging assist detection of focal cortical dysplasia in extra-temporal partial epilepsy. Ann Neurol 2001;49:770-75

21. Colombo N, Tassi L, Galli C, et al. Focal cortical dysplasias: MR imaging, histopathologic, and clinical correlations in surgically treated patients with epilepsy. AJNR Am J Neuroradiol 2003;24:724-33

22. Barkovich AJ, Kuzniecky RI, Bollen AW, et al. Focal transmantle dysplasia: a specific malformation of cortical development. Neurology 1997;49:1148-52

23. Duncan JS. Imaging in the surgical treatment of epilepsy. Nat Rev Neurol 2010;6:537-50

24. Chamberlain WA, Cohen ML, Gyure KA, et al. Interobserver and intraobserver reproducibility in focal cortical dysplasia (malformations of cortical development). Epilepsia 2009;50:2593-98

25. Colombo N, Salamon N, Raybaud C, et al. Imaging of malformations of cortical development. Epileptic Disord 2009;11:194-205

26. Besson P, Andermann F, Dubeau F, et al. Small focal cortical dysplasia lesions are located at the bottom of a deep sulcus. Brain 2008;131:3246-55

27. Lasjaunias P, Manelfe C, Terbrugge K, et al. Endovascular treatment of cerebral arteriovenous malformations. Neurosurg Rev 1986;9:265-75

28. Widjaja E, Otsubo H, Raybaud C, et al. Characteristics of MEG and MRI between Taylor's focal cortical dysplasia (type II) and other cortical dysplasia: surgical outcome after complete resection of MEG spike source and MR lesion in pediatric cortical dysplasia. Epilepsy Res 2008;82:147-55

29. Bronen RA, Spencer DD, Fulbright RK. Cerebrospinal fluid cleft with cortical dimple: MR imaging marker for focal cortical dysgenesis. Radiology 2000; 214:657-63

30. Regis J, Tamura M, Park MC, et al. Subclinical abnormal gyration pattern, a potential anatomic marker of epileptogenic zone in patients with magnetic resonance imaging-negative frontal lobe epilepsy. Neurosurgery 2011;69: $80-93$

31. McGonigal A, Bartolomei F, Regis J, et al. Stereoelectroencephalography in presurgical assessment of MRI-negative epilepsy. Brain 2007;130:3169-83

32. Chapman K, Wyllie E, Najm I, et al. Seizure outcome after epilepsy surgery in patients with normal preoperative MRI. J Neurol Neurosurg Psychiatry 2005;76:710-13

33. Bernasconi A, Bernasconi N, Bernhardt BC, et al. Advances in MRI for 'cryptogenic' epilepsies. Nat Rev Neurol 2011;7:99-108

34. Wagner J, Weber B, Urbach $\mathrm{H}$, et al. Morphometric MRI analysis improves detection of focal cortical dysplasia type II. Brain 2011;134:2844-54

35. Knake S, Triantafyllou C, Wald LL, et al. 3T phased array MRI improves the presurgical evaluation in focal epilepsies: a prospective study. Neurology 2005; 65:1026-31 